\title{
Phase- and Polarization-Controlled Two-Photon Rabi Oscillation of the Biexciton State in a Semiconductor Quantum Dot
}

\author{
Erlin Sun, Donghai Feng, and Tianqing Jia \\ State Key Laboratory of Precision Spectroscopy, East China Normal University, Shanghai 200062, China \\ Correspondence should be addressed to Donghai Feng; dhfeng@phy.ecnu.edu.cn
}

Received 29 May 2013; Revised 1 November 2013; Accepted 11 November 2013; Published 19 January 2014

Academic Editor: Sergio E. Ulloa

Copyright (C) 2014 Erlin Sun et al. This is an open access article distributed under the Creative Commons Attribution License, which permits unrestricted use, distribution, and reproduction in any medium, provided the original work is properly cited.

\begin{abstract}
Under a degenerate two-photon resonant excitation, the Rabi oscillation of the four-level biexciton system in a semiconductor quantum dot is theoretically investigated. The influence of the laser phases on the state manipulation is modeled and numerically calculated. Due to the interference between different excitation paths, the laser phase plays an important role and can be utilized as an alternate control knob to coherently manipulate the biexciton state. The phase control can be facilely implemented by changing the light polarization via a quarter-wave plate.
\end{abstract}

\section{Introduction}

The characteristic of atomic-like discrete energy levels, together with the ability of integration in solid-state devices, makes quantum dots (QDs) very promising for solid-state implementations of quantum information processing (QIP) $[1,2]$. Therein, the biexciton system, consisting of two mutually coupled excitons in QDs, has attracted much interest as it carries prospects in realization of two-bit quantum logic gates $[3,4]$ and in generation of entangled photon pairs $[5,6]$.

Coherent manipulation of quantum states is a basic issue for QIP. Among the state manipulation, Rabi oscillation is a fundamental phenomenon by coherent optical coupling, which provides a direct control to the state population and coherence of a quantum system. Rabi oscillations have been well studied theoretically and experimentally both for exciton and biexciton systems in a QD [3, 4, 7-12]. Rabi oscillations are typically measured by controlling the pulse-area, via the field intensity (excitation power) of the incident laser pulse.

Excitation for the biexciton system is in a closed-loop four-level scheme shown in Figure 1, where the ground state $|g\rangle$ is coupled in a V-type structure to intermediate exciton doublet states $|x\rangle$ and $|y\rangle$, which are themselves coupled to a common excited biexciton state $|b\rangle$ in a $\Lambda$-type structure. The biexciton state can be excited via $|g\rangle \rightarrow|x\rangle \rightarrow|b\rangle$ by $\Pi_{x}$ linearly polarized photons or $|g\rangle \rightarrow|y\rangle \rightarrow|b\rangle$ by $\Pi_{y}$ linearly polarized photons. Previous studies related to biexciton were normally done by selecting one of the two excitation paths with proper polarized light. Herein biexciton Rabi oscillation is simply a function of the field intensity of the light. Now, supposing under the excitation of a light with both $\Pi_{x}$ and $\Pi_{y}$ components, then both the two excitation paths will be activated. In a closed-loop $\diamond$ configuration, the two excitation paths can interfere, and the state control is dependent on the relative phase between the two paths [13$16]$.

In this paper, we investigate Rabi oscillations of the biexciton in a semiconductor QD, based on a resonant degenerate two-photon excitation $[17,18]$ with both $\Pi_{x}$ and $\Pi_{y}$ components. In addition to the field strength of the excitation light, the relative phase between $\Pi_{x}$ and $\Pi_{y}$ components (and consequently the light polarization) is shown to play an important role on the state manipulation and can serve as an alternate control knob to coherently manipulate the biexciton system.

\section{The Model}

A picosecond pulse laser with a central photon energy of $\hbar \omega$, which is equal to half the biexciton energy, is used for the 


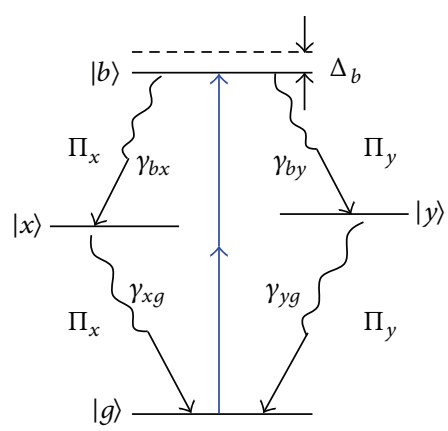

FIGURE 1: Scheme of optical transitions for the biexciton system in a quantum dot. See text for explanation.

excitation. The light has both $\Pi_{x}$ and $\Pi_{y}$ polarized components of equal amplitudes. The phase difference $\Phi$ between the two components is set as an adjustable parameter. The time evolution of the density matrix $\rho$ for the biexciton system is determined by the master equation

$$
\frac{\partial}{\partial t} \rho=\frac{1}{i \hbar}[H(t), \rho]+L(\rho)
$$

where

$$
\begin{gathered}
H(t)=\hbar \omega_{x}|x\rangle\left\langle x\left|+\hbar \omega_{y}\right| y\right\rangle\left\langle y\left|+\hbar \omega_{b}\right| b\right\rangle\langle b| \\
+\frac{\hbar \Omega(t)}{2}\left\{e ^ { - i \omega t } \left(|x\rangle\langle g|+| b\rangle\left\langle x\left|+e^{i \Phi}\right| y\right\rangle\langle g|\right.\right. \\
\left.\left.+e^{i \Phi}|b\rangle\langle y|\right)+ \text { H.c. }\right\},
\end{gathered}
$$

and Lindblad term $L(\rho)[19,20]$ describes the relaxation process of excitons and biexcitons, which may be caused by spontaneous emission and carrier-phonon scattering. In this paper, we only consider the spontaneous emission part for simplicity. Then

$$
L(\rho)=\sum_{i j} \frac{\gamma_{i j}}{2}\left(2 \sigma_{i j}^{\dagger} \rho \sigma_{i j}-\sigma_{i j} \sigma_{i j}^{\dagger} \rho-\rho \sigma_{i j} \sigma_{i j}^{\dagger}\right),
$$

where $\sigma_{i j} \equiv|i\rangle\langle j|$ denotes the spontaneous emission transition from $i$ to $j$ including $\sigma_{b x}, \sigma_{b y}, \sigma_{x g}$, and $\sigma_{y g} \cdot \gamma_{i j}$ describes the corresponding decay rates.

In the rotating frame with the canonical transformation defined by the unitary operator $U=e^{i \omega t|x\rangle\langle x|+i \omega t| y\rangle\langle y|+2 i \omega t| b\rangle\langle b|}$, $H(t)$ can be rewritten as

$$
\begin{aligned}
\widetilde{H}(t)= & U H U^{\dagger}+i \hbar \frac{d U}{d t} U^{\dagger} \\
= & \sum_{j=x, y, b} \hbar \delta_{j}|j\rangle\langle j|+\frac{\hbar}{2} \Omega(t) \\
& \times\left(|x\rangle\langle g|+| b\rangle\left\langle x\left|+e^{i \Phi}\right| y\right\rangle\langle g|\right. \\
& \left.+e^{i \Phi}|b\rangle\langle y|+\text { H.c. }\right) .
\end{aligned}
$$

The detunings $\delta_{x, y}$ and $\delta_{b}$ in (4) are given by $\delta_{x}=\omega_{x}-$ $\omega=\left(\Delta_{b}-\delta\right) / 2, \delta_{y}=\omega_{y}-\omega=\left(\Delta_{b}+\delta\right) / 2$, and $\delta_{b}=$ $\omega_{b}-2 \omega=0$, where $\Delta_{b}$ and $\delta$ are the biexciton binding energy and exciton doublet splitting energy, respectively. $\Omega$ is the time-dependent Rabi frequency of the pulse. Assuming using a Gaussian shape pulse, $\Omega(t)=\Theta /(\tau \sqrt{\pi}) \exp \left(-t^{2} / \tau^{2}\right)$, where $\Theta$ is the pulse area and $\tau$ is a parameter for the pulse duration. $\tau=\tau_{\mathrm{FWHM}} /(2 \sqrt{\ln 2})$, where $\tau_{\mathrm{FWHM}}$ is the pulse duration defined by the full width at half maximum of the intensity.

Similar to [13], the optical Bloch equations then have the form

$$
\begin{aligned}
& \dot{\rho}_{g g}=i \frac{\Omega}{2}\left(\rho_{g x}-\rho_{x g}\right)+i \frac{\Omega}{2}\left(e^{i \Phi} \rho_{g y}-e^{-i \Phi} \rho_{y g}\right) \\
& +\gamma_{x g} \rho_{x x}+\gamma_{y g} \rho_{y y}, \\
& \dot{\rho}_{x x}=-i \frac{\Omega}{2}\left(\rho_{g x}-\rho_{x g}\right)+i \frac{\Omega}{2}\left(\rho_{x b}-\rho_{b x}\right) \\
& +\gamma_{b x} \rho_{b b}-\gamma_{x g} \rho_{x x}, \\
& \dot{\rho}_{y y}=-i \frac{\Omega}{2}\left(e^{i \Phi} \rho_{g y}-e^{-i \Phi} \rho_{y g}\right)+i \frac{\Omega}{2}\left(e^{i \Phi} \rho_{y b}-e^{-i \Phi} \rho_{b y}\right) \\
& +\gamma_{b y} \rho_{b b}-\gamma_{y g} \rho_{y y}, \\
& \rho_{b b}=1-\rho_{g g}-\rho_{x x}-\rho_{y y} \text {, } \\
& \dot{\rho}_{g x}=\left(i \delta_{x}-\frac{\gamma_{x g}}{2}\right) \rho_{g x}-i \frac{\Omega}{2}\left(\rho_{x x}-\rho_{g g}\right) \\
& -i \frac{\Omega}{2} e^{-i \Phi} \rho_{y x}+i \frac{\Omega}{2} \rho_{g b}, \\
& \dot{\rho}_{g y}=\left(i \delta_{y}-\frac{\gamma_{y g}}{2}\right) \rho_{g x}-i \frac{\Omega}{2} e^{-i \Phi}\left(\rho_{y y}-\rho_{g g}\right) \\
& -i \frac{\Omega}{2} \rho_{x y}+i \frac{\Omega}{2} e^{i \Phi} \rho_{g b}, \\
& \dot{\rho}_{x b}=\left(-i\left(\delta_{x}-\delta_{b}\right)-\frac{\gamma_{x g}+\gamma_{b x}+\gamma_{b y}}{2}\right) \rho_{x b} \\
& -i \frac{\Omega}{2}\left(\rho_{b b}-\rho_{x x}\right)-i \frac{\Omega}{2} \rho_{g b}+i \frac{\Omega}{2} e^{-i \Phi} \rho_{x y}, \\
& \dot{\rho}_{y b}=\left(-i\left(\delta_{y}-\delta_{b}\right)-\frac{\gamma_{y g}+\gamma_{b x}+\gamma_{b y}}{2}\right) \rho_{y b} \\
& -i \frac{\Omega}{2} e^{-i \Phi}\left(\rho_{b b}-\rho_{y y}\right)-i \frac{\Omega}{2} e^{i \Phi} \rho_{g b}+i \frac{\Omega}{2} \rho_{y x}, \\
& \dot{\rho}_{g b}=\left(i \delta_{b}-\frac{\gamma_{b x}+\gamma_{b y}}{2}\right) \rho_{g b}-i \frac{\Omega}{2} \rho_{x b} \\
& -i \frac{\Omega}{2} e^{-i \Phi} \rho_{y b}+i \frac{\Omega}{2} \rho_{g x}+i \frac{\Omega}{2} e^{-i \Phi} \rho_{g y}, \\
& \dot{\rho}_{x y}=\left[-i\left(\delta_{x}-\delta_{y}\right)-\frac{\gamma_{x g}+\gamma_{y g}}{2}\right] \rho_{x y} \\
& -i \frac{\Omega}{2}\left(\rho_{g y}+\rho_{b y}-e^{-i \Phi} \rho_{x g}-e^{i \Phi} \rho_{x b}\right),
\end{aligned}
$$




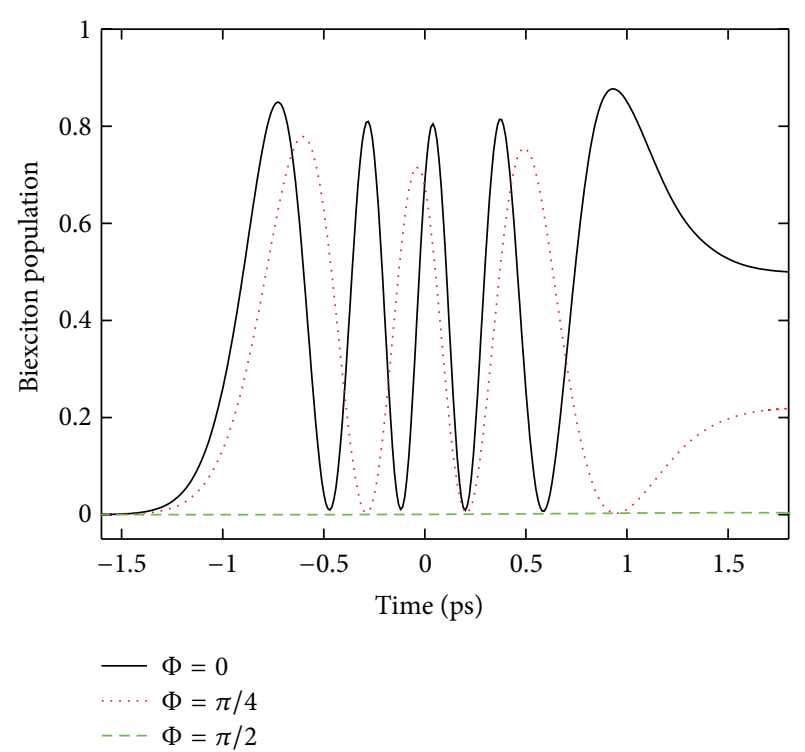

FIGURE 2: Biexciton Rabi oscillations within the pulse duration of 1 ps with different relative phase $\Phi$ at the pulse area of $15 \pi$. The biexciton binding energy $\Delta_{b}=20 \mathrm{meV}$, and the exciton doublet splitting energy $\delta=0.15 \mathrm{meV}$.

and $\rho_{j i}=\left(\rho_{i j}\right)^{*}$. In the following, the diagonal elements will be referred as "populations" in the corresponding states and the off-diagonal elements as the coherence between the related two levels; for example, $\rho_{g b}$ is ground-biexciton coherence.

\section{Results and Discussion}

The optical Bloch equations can be numerically solved. The parameters for the calculations are $\tau=1 \mathrm{ps}, \Delta_{b}=20 \mathrm{meV}$, $\delta=0.15 \mathrm{meV}$, and $\gamma_{b x}=\gamma_{b y}=\gamma_{x g}=\gamma_{y g}=1 / 200 \mathrm{ps}^{-1}$, which are taken as comparable to the experimental values for II-VI CdSe/ZnSe QDs [17, 18]. In the following, we will only figure out the data of the biexciton population $\rho_{b b}$ and groundbiexciton coherence $\rho_{g b}$. The elements in the density matrix related to one photon process are normally small due to nonresonant excitations. The coherence within the excitonic doublet, $\rho_{x y}$, is also small as it is related to a nonresonant twophoton excitation process.

Figure 2 shows the temporal evolution of biexciton populations with different relative phase $\Phi$ at a pulse area $\Theta=15 \pi$. For $\Phi=0$, five cycles of population oscillations are clearly resolved. The occupation oscillates with a timedependent frequency, which is a result from the Gaussianshape temporal distribution of the driving field strength. For times nearer the pulse maximum at $t=0$, the oscillation develops faster and the period appears shorter. For $\Phi=\pi / 4$, only about three cycles of oscillations exist. For $\Phi=\pi / 2$, there is no oscillation, and the biexciton population is near to zero within the whole pulse duration. From Figure 2, one can see that the relative phase $\Phi$ dramatically affects both the oscillation evolution and the final population value at the end of the pulse.
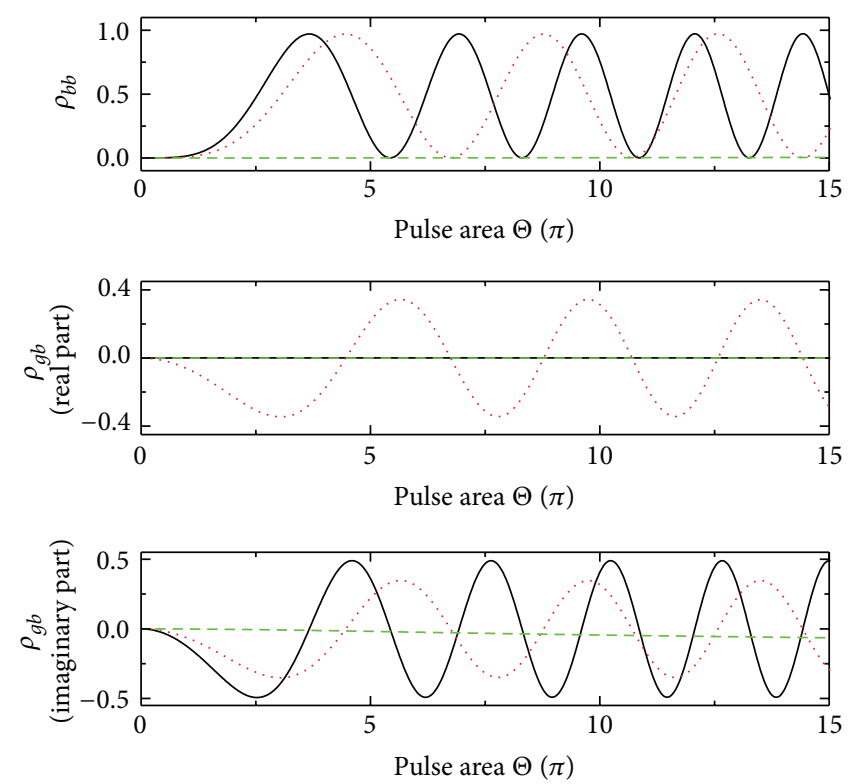

$$
\begin{aligned}
-\Phi & =0 \\
\cdots \cdots \Phi & =\pi / 4 \\
---\Phi & =\pi / 2
\end{aligned}
$$

FIGURE 3: Rabi oscillations as a function of pulse area $\Theta$ with different relative phase $\Phi . \rho_{b b}$ : Biexciton population, $\rho_{g b}$ : Groundbiexciton coherence. The biexciton binding energy $\Delta_{b}=20 \mathrm{meV}$, and the exciton doublet splitting energy $\delta=0.15 \mathrm{meV}$.

Figure 3 shows the biexciton population and groundbiexciton coherence at the end of the excitation pulse as a function of pulse area with different phases. The values oscillate as the pulse area increases. Different from normal Rabi oscillations induced by one photon excitation, the oscillations in Figure 3 are not strictly periodic and the system develops slowly at low pulse area. This is typical for two-photon excitation process $[7,8]$, as both biexciton populations and ground-biexciton coherences are proportional to the square of the pulse intensity, that is, $\Theta^{4}$, at low excitation power.

For $\Phi=\pi / 2$, the excitation paths between $|g\rangle \rightarrow|x\rangle \rightarrow$ $|b\rangle$ and $|g\rangle \rightarrow|y\rangle \rightarrow|b\rangle$ destructively interfere, making the biexciton population and ground-biexciton coherence (both the real and imaginary parts) almost zero (see green dash lines in Figure 3). However, there exists a small deviation from zero, which increases with the pulse area. For the biexciton population, the deviation is up to the order of magnitude of $10^{-3}$ using the pulse area data shown in Figure 3. Nonzero biexciton population results from the asymmetric excitation between the two excitation paths due to the nondegenerate exciton doublet states.

For $\Phi=0$, the real part of the ground-biexciton coherence $\rho_{g b}$ is almost zero (enshrouded by the green dash line for $\Phi=\pi / 2$ ). This phenomenon is similar to the fact that the real part of the coherence between the related two levels is zero for a resonantly driven two-level system. The small deviation from zero (up to the order of magnitude of $10^{-4}$ ) is due to the scattering by the nonresonant excitation of the exciton doublet states. 

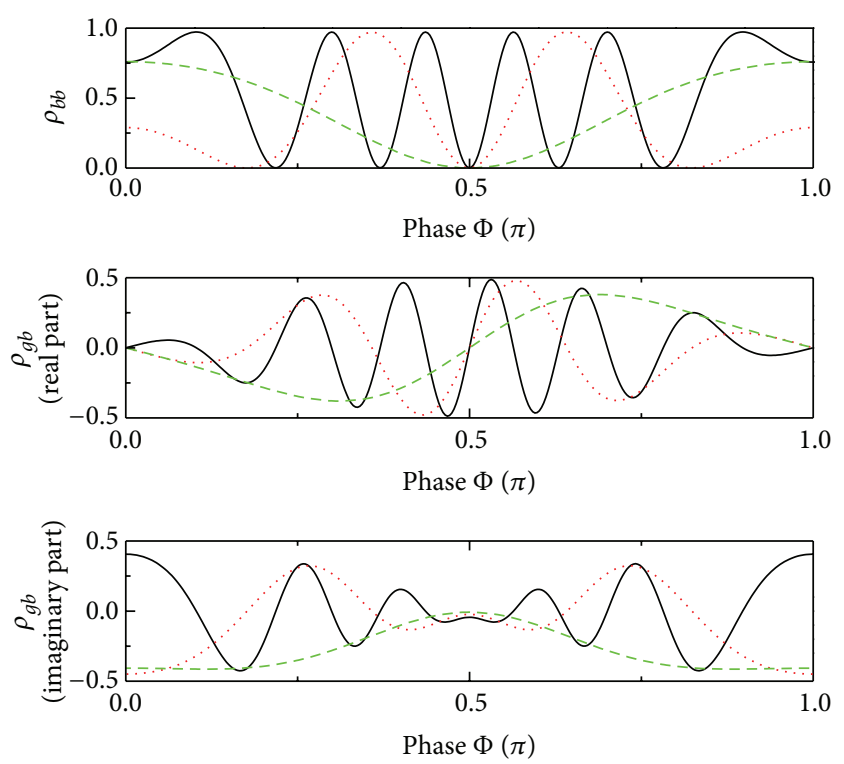

$$
\begin{aligned}
-\Theta & =10 \pi \\
\ldots . \Theta & =6 \pi \\
---\Theta & =3 \pi
\end{aligned}
$$

FIgURE 4: Rabi oscillations as a function of relative phase $\Phi$ with different pulse area $\Theta . \rho_{b b}$ : Biexciton population, $\rho_{g b}$ : Groundbiexciton coherence. The biexciton binding energy $\Delta_{b}=20 \mathrm{meV}$, and the exciton doublet splitting energy $\delta=0.15 \mathrm{meV}$.

Figure 4 shows the biexciton population and groundbiexciton coherence at the end of the excitation pulse as a function of laser phase with different pulse area. The system could oscillate several cycles for large pulse area within a phase period of $\pi$. Larger pulse area corresponds to more cycles of oscillations. As already mentioned for Figure 3, small deviation from zero at $\Phi=\pi / 2$ is resulted from the asymmetric exciton excitation (e.g., see the imaginary part of ground-biexciton coherence, which appears more clearly. The ground-biexciton coherence is nonzero and its absolute values increase with pulse area for $\Phi=\pi / 2$ ).

Figures 2-4 are calculated for II-VI semiconductor QDs which normally has relatively large biexciton binding energy and exciton doublet splitting energy. For III-V QDs, these values are normally much small, for example, $\Delta_{b} \sim 3 \mathrm{meV}$ and $\delta \sim 0.03 \mathrm{meV}$ for InGaAs QDs [7, 12, 21]. For smaller biexciton binding energy $\Delta_{b}$, a laser pulse with a photon energy being equal to half the biexciton energy will pump the exciton states more effectively as compared to the case with larger $\Delta_{b}$, since the detunings have the form of $\delta_{x}=$ $\left(\Delta_{b}-\delta\right) / 2$ and $\delta_{y}=\left(\Delta_{b}+\delta\right) / 2$ as mentioned above. Furthermore, small exciton doublet splitting of energy of $30 \mu \mathrm{eV}$ can be neglected under a picosecond pulse excitation [7]. Figure 5 shows the calculated phase dependence of biexciton populations and ground-biexciton coherences at the end of the excitation pulse with $\Delta_{b}=3 \mathrm{meV}$ and $\delta=$ $0.03 \mathrm{meV}$. Compared with Figure 4 , there are more cycles of oscillations in Figure 5 under the same excitation pulse and
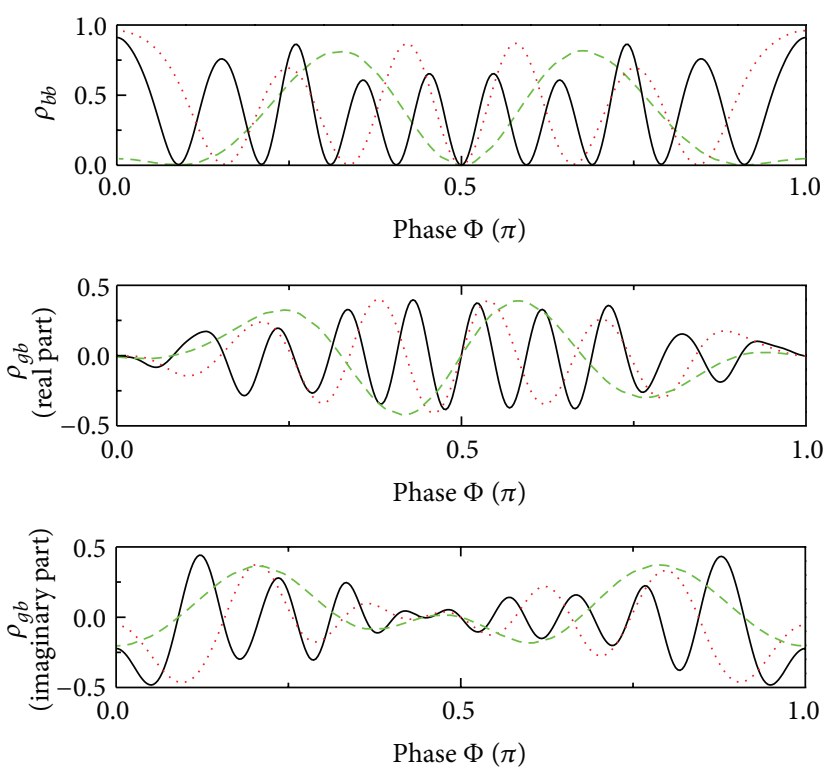

$$
\begin{aligned}
-\Theta & =10 \pi \\
\cdots \Theta & =6 \pi \\
---\Theta & =3 \pi
\end{aligned}
$$

Figure 5: Rabi oscillations as a function of relative phase $\Phi$ with different pulse area $\Theta$. $\rho_{b b}$ : Biexciton population, $\rho_{g b}$ : Groundbiexciton coherence. The biexciton binding energy $\Delta_{b}=3 \mathrm{meV}$, and the exciton doublet splitting energy $\delta=0.03 \mathrm{meV}$. The other parameters are the same as for Figure 4.

the same decay rates $\gamma_{i j}$. The reason is that smaller detunings $\delta_{x, y}$ make the system more coupled to the excitation light.

The phase control of two-photon Rabi oscillation for the biexciton system can be easily implemented experimentally. Zero phase between $\Pi_{x}$ and $\Pi_{y}$ photon corresponds to a 45-degree linearly polarized light, while the phase difference of $\pi / 2$ denotes a circularly polarized light. And the phase difference between 0 and $\pi / 2$ describes an elliptically polarized light. By using a quarter-wave plate, the light from linear polarization to elliptic and to circular polarization can be facilely obtained. The numerical results in Figures $2-5$ are consistent with the fact that a circularly polarized light cannot excite the biexciton if the exciton doublet states are degenerate, according to polarization selection rules.

\section{Conclusions}

The two-photon Rabi oscillation of the biexciton state in a semiconductor quantum dot has been investigated theoretically. In a closed-loop $\diamond$ configuration, such as the biexciton system, not only the field strength but also the laser phase is shown to influence the Rabi oscillation. Apart from the usually used pulse area, that is, field strength, the laser phase is promising as an alternate control knob to coherently manipulate the biexciton state, which can be facilely implemented by changing the light polarization via a quarter-wave plate. 


\section{Conflict of Interests}

The authors declare that there is no conflict of interests regarding the publication of this paper.

\section{Acknowledgments}

This work was partly supported by National Key Project for Basic Research of China (Grant no. 2010CB923203), National Natural Science Foundation of China (Grants nos. 10904038, 11104078, and 11374099), and Shanghai Municipal Science and Technology Commission (Grants nos. 10PJ1403400 and 11JC1403500).

\section{References}

[1] D. Loss and D. P. Divincenzo, "Quantum computation with quantum dots," Physical Review A, vol. 57, no. 1, pp. 120-126, 1998.

[2] F. Henneberger and O. Benson, Semiconductor Quantum Bits, World Scientific, Singapore, 2008.

[3] X. Li, Y. Wu, D. Steel et al., "An all-optical quantum gate in a semiconductor quantum dot," Science, vol. 301, no. 5634, pp. 809-811, 2003.

[4] S. J. Boyle, A. J. Ramsay, F. Bello et al., "Two-qubit conditional quantum-logic operation in a single self-assembled quantum dot," Physical Review B, vol. 78, no. 7, Article ID 075301, 6 pages, 2008.

[5] O. Benson, C. Santori, M. Pelton, and Y. Yamamoto, "Regulated and entangled photons from a single quantum dot," Physical Review Letters, vol. 84, no. 11, pp. 2513-2516, 2000.

[6] S. Schumacher, J. Förstner, A. Zrenner et al., "Cavity-assisted emission of polarization-entangled photons from biexcitons in quantum dots with fine-structure splitting," Optics Express, vol. 20, no. 5, pp. 5335-5342, 2012.

[7] S. Stufler, P. Machnikowski, P. Ester et al., "Two-photon Rabi oscillations in a single $\operatorname{In}_{x} \mathrm{Ga}_{1-x}$ As GaAs quantum dot," Physical Review B, vol. 73, no. 12, Article ID 125304, 7 pages, 2006.

[8] P. Machnikowski, "Theory of two-photon processes in quantum dots: coherent evolution and phonon-induced dephasing," Physical Review B, vol. 78, no. 19, Article ID 195320, 10 pages, 2008.

[9] S. J. Boyle, A. J. Ramsay, A. M. Fox, M. S. Skolnick, A. P. Heberle, and M. Hopkinson, "Beating of exciton-dressed states in a single semiconductor InGaAs/GaAs quantum dot," Physical Review Letters, vol. 102, no. 20, Article ID 207401, 4 pages, 2009.

[10] H. Kamada, H. Gotoh, J. Temmyo, T. Takagahara, and H. Ando, "Exciton Rabi oscillation in a single quantum dot," Physical Review Letters, vol. 87, no. 24, Article ID 246401, 4 pages, 2001.

[11] T. H. Stievater, X. Li, D. G. Steel et al., "Rabi oscillations of excitons in single quantum dots," Physical Review Letters, vol. 87, no. 13, Article ID 133603, 4 pages, 2001.

[12] A. Zrenner, E. Beham, S. Stufler, F. Findeis, M. Bichler, and G. Abstreiter, "Coherent properties of a two-level system based on a quantum-dot photodiode," Nature, vol. 418, no. 6898, pp. 612$614,2002$.

[13] G. Morigi, S. Franke-Arnold, and G.-L. Oppo, "Phasedependent interaction in a four-level atomic configuration," Physical Review A, vol. 66, no. 5, Article ID 053409, 9 pages, 2002.
[14] V. S. Malinovsky and I. R. Sola, "Phase-controlled collapse and revival of entanglement of two interacting qubits," Physical Review Letters, vol. 96, no. 5, Article ID 050502, 4 pages, 2006.

[15] V. S. Malinovsky and I. R. Sola, "Quantum phase control of entanglement," Physical Review Letters, vol. 93, no. 19, Article ID 190502, 4 pages, 2004.

[16] M. C. Stowe, A. Pe'er, and J. Ye, "Control of four-level quantum coherence via discrete spectral shaping of an optical frequency comb," Physical Review Letters, vol. 100, no. 20, Article ID 203001, 4 pages, 2008.

[17] I. A. Akimov, J. T. Andrews, and F. Henneberger, "Stimulated emission from the biexciton in a single self-assembled II-VI quantum dot," Physical Review Letters, vol. 96, no. 6, Article ID 067401, 4 pages, 2006.

[18] T. Flissikowski, A. Betke, I. A. Akimov, and F. Henneberger, "Two-photon coherent control of a single quantum dot," Physical Review Letters, vol. 92, no. 22, Article ID 227401, 4 pages, 2004.

[19] G. Lindblad, "On the generators of quantum dynamical semigroups," Communications in Mathematical Physics, vol. 48, no. 2, pp. 119-130, 1976.

[20] H. Y. Hui and R. B. Liu, "Proposal for geometric generation of a biexciton in a quantum dot using a chirped pulse," Physical Review B, vol. 78, no. 15, Article ID 155315, 6 pages, 2008.

[21] J. M. Villas-Bôas, S. E. Ulloa, and A. O. Govorov, "Spin polarized photocurrent from quantum dots," Physical Review B, vol. 75, no. 15, Article ID 155334, 7 pages, 2007. 

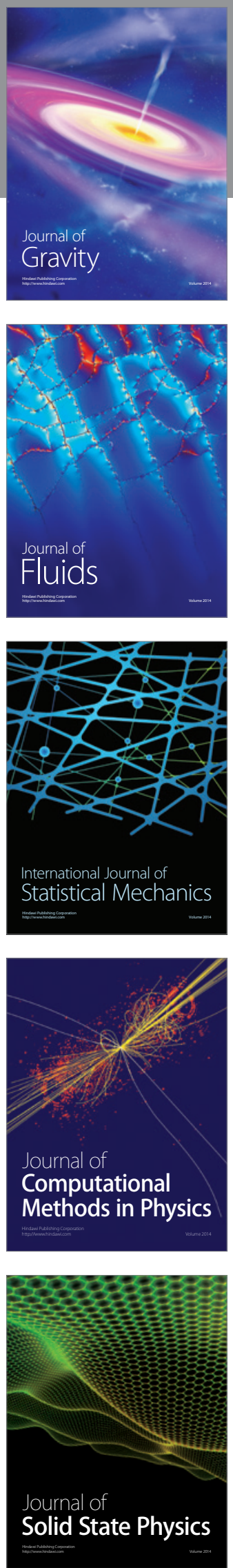

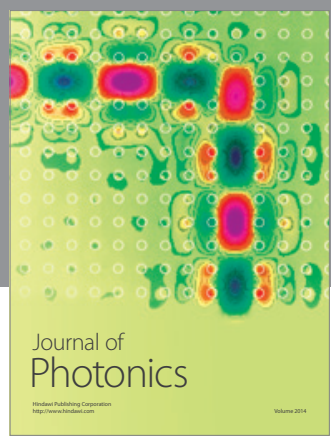

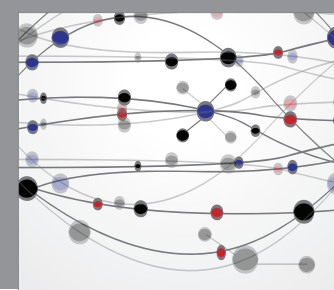

The Scientific World Journal

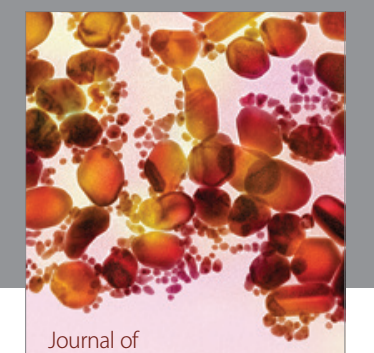

Soft Matter
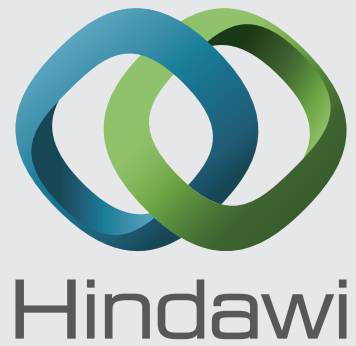

Submit your manuscripts at

http://www.hindawi.com
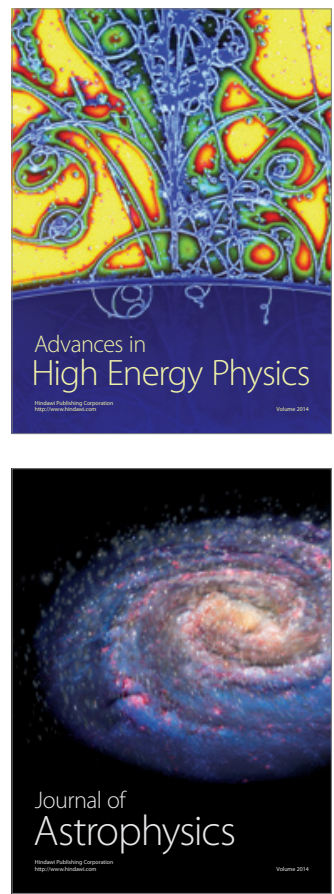
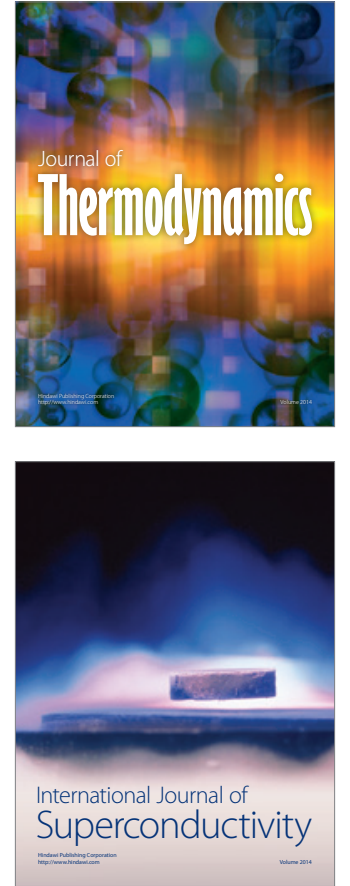
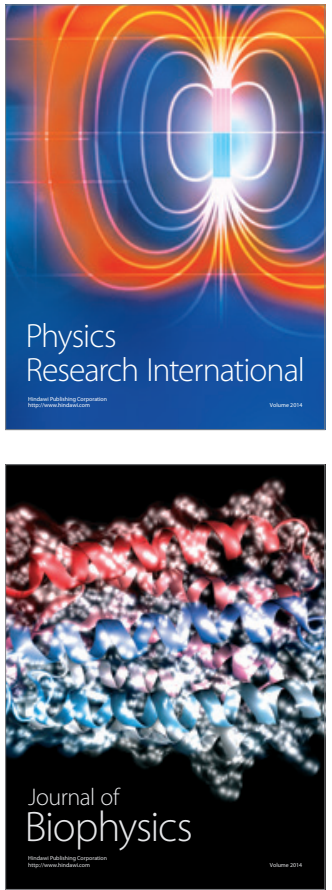
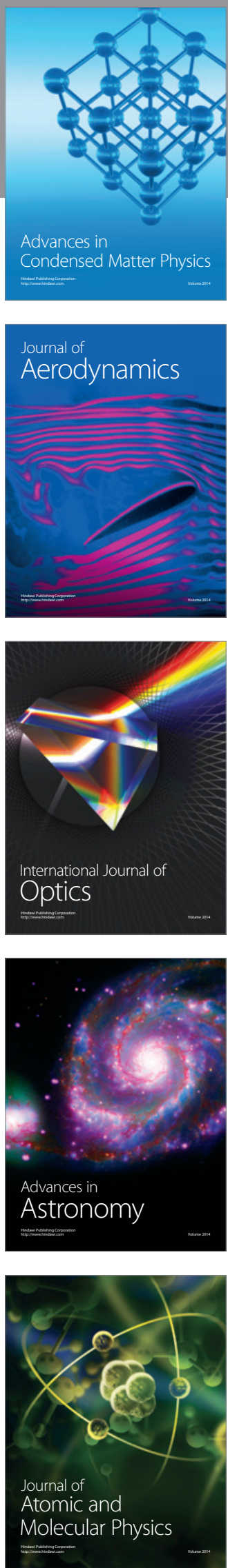